\title{
Body temperature control in fever modeling after preliminary injection of glutamate receptors ligands into the solitary tract nucleus
}

\author{
Stanislav V. Koulchitsky ${ }^{1,2}$, Svetlana G. Pashkevich ${ }^{1}$, Nastassia M. Navasiolava ${ }^{1,3}$, \\ Marc-Antoine Custaud ${ }^{3}$, Vladimir A. Kulchitsky ${ }^{1}$ \\ ${ }^{1}$ Institute of Physiology of NAS of Belarus, Minsk, Belarus \\ ${ }^{2}$ Laboratory of Pharmacology and GIGA Neurosciences, University of Liège, Liège, Belgium \\ ${ }^{3}$ The National Centre for Scientific Research, Joint Research Unit 6214 - The French National Institute of Health and Medical \\ Research, Joint Research Unit 1083 \\ Email: stas.koulchitsky@gmail.com
}

Received 20 January 2013; revised 5 March 2013; accepted 9 April 2013

Copyright (C) 2013 Stanislav V. Koulchitsky et al. This is an open access article distributed under the Creative Commons Attribution License, which permits unrestricted use, distribution, and reproduction in any medium, provided the original work is properly cited.

\begin{abstract}
Deep body temperature of 57 male Wistar rats was measured in response to systemic (i.v. or i.p.) injection of Escherichia coli lipopolysaccharide (LPS). Animals were preliminarily ( 4 weeks before the experiment) treated with microinjections of the toxic dose of glutamate receptors agonists or vehicle into solitary tract nucleus or caudal part of ventrolateral medulla. Microinjections of the glutamate receptors agonists provoked a local destruction of brain tissue, which was accompanied by the significant transformation (amplification or attenuation) of temperature response to the systemic injection of $3 \mu \mathrm{g} / \mathrm{kg} \mathrm{E}$. coli LPS. Animals treated with vehicle did not demonstrate significant changes in their response to LPS. Obtained data testify that impairment of central nervous structure involved in the regulation and maintenance of deep body temperature is accompanied by atypical development of $E$. coli LPS-induced fever.
\end{abstract}

Keywords: Escherichia coli; Endotoxin; Brainstem; Fever; Control Body Temperature; Defense Reflexes

\section{INTRODUCTION}

According to current data, translocation of bacterial microflora and endotoxins from the gut lumen into the internal milieu may happen after various operations in the abdominal cavity or the action of different extreme factors on the organism [1-3]. This initiates various types of protective reactions at systemic and local levels. Penetration of intestinal microflora into the lymph and systemic circulation can be followed by changes in nervous, immune and endocrine systems functioning [1,3-6] that triggers the development of protective reactions, and fever is one of them $[3,4]$.

In experimental conditions, this process is modeled using bacterial lipopolysaccharides (LPS). Some previous research suggested that primary fever-initiating signal from systemic LPS comes through the peripheral vagal afferents [7-12]. Transection of the hepatic branch of the vagus markedly attenuated nociceptive and temperature responses to endotoxin $[13,14]$. However, recent findings demonstrate that LPS or IL-1 $\beta$ induced fever does not necessarily require vagal signaling, since vagotomy does not change fever progress with intravenous injection of $\mathrm{PGE}_{2}$ [5]. This apparent contradiction can be explained by the hypothesis that effect of vagotomy is indirect and due to the destructive changes in the brainstem by vagus transection. Central degeneration after the peripheral vagotomy was verified by morphologists in different animals [15]. Particularly, destructive processes were shown in the primary vagal projections 4 weeks after the vagotomy [15].

Nucleus of the solitary tract (NST) - the primary afferent projection zone of the vagus and glossopharyngeal nerves - is one of the key relay areas in the receiving afferent signals from visceral thermoreceptors. From the NST the impulses flow to hypothalamic and bulbar nuclei. One of the important bulbar areas, receiving inputs from NST is ventral medulla, which transfers the signals in rostral and caudal directions to the hypothalamic nuclei and the sympathetic preganglionic neurons of the spinal cord, respectively [16-20]. This system reciprocally interacts with the higher brain regions integrating cognitive and emotional signals with the information 
coming from the periphery, contributing to the control of energy balance [21]. Neurons possessing glutamate receptors are shown to be involved in the analysis of nociceptive and thermal signaling [21-26].

In the present study we used local neurotoxin injections to partially destroy some of neuronal populations, which are known to degenerate after the subdiaphragmatic vagotomy. Namely, neurons in the NST and the caudal part of the VLM. The question was whether this central intervention can affect the thermoregulatory reaction to the systemic injection of $E$. coli lipopolysaccharide?

\section{MATERIALS AND METHODS}

Experiments were performed using male Wistar rats $(\mathrm{n}=$ 57) weighing $250-300 \mathrm{~g}$. All animals had food and water ad libitum. All surgical procedures were made under ketamine-xylazine-acepromazine (55.6 - $5.5-1.1 \mathrm{mg} / \mathrm{kg}$, respectively, intraperitoneally) anesthesia. Broad-spectrum antibiotic was injected simultaneously (Enrofloxacin, $0.1 \mu \mathrm{g} / \mathrm{kg}$, intramuscularly).

Intracerebral microinjections were made four weeks before the experiments. The rat's head was fixed in stereotaxic device SEJ - 5 (Experimental workshops of the Institute of Physiology named after A. A. Bogomoletz, National Academy of Sciences of Ukraine, Kiev, Ukraine) to install lambda and bregma at the same level. Animals' skin was treated with 5\% iodine solution, and subcutaneous injection of $2 \%$ novocaine solution was made. Soft tissues of cranium vault were dissected, the periosteum removed (after additional injection of $2 \%$ novocaine subperiosteally). Special attention was paid to perform a careful haemostasis. The aperture $1 \mathrm{~mm}$ in diameter was made in skull bones according to stereotaxic coordinates [27] using a microdrill. Dura mater was gently dissected. A glass micropipette (with tip diameter of $30-50 \mu \mathrm{m}$ ) was introduced through the trepanation aperture into the brain tissue using micromanipulator. Microinjections were made using a nanoliter micropump (W-P Instruments, Inc. 1400, USA). Different groups of animals received microinjections into rostral $(3.5 \mathrm{~mm}$ caudal to interaural line, $0 \mathrm{~mm}$ lateral to midline and $8 \mathrm{~mm}$ in depth from the skull surface) or caudal ( $4.5 \mathrm{~mm}$ caudal to interaural line, $0 \mathrm{~mm}$ lateral to midline and $8 \mathrm{~mm}$ in depth from the skull surface) parts of commissural department of NST or bilaterally into caudal parts of VLM $(3.5 \mathrm{~mm}$ caudal to interaural line, $2 \mathrm{~mm}$ lateral to midline and $9.5 \mathrm{~mm}$ in depth from the skull surface) [27]. The injected solutions represented 1) $100 \mathrm{nl}$ of saline $(0.9 \% \mathrm{NaCl})$; 2) $0.5 \mu \mathrm{g}$ of kainic acid in $100 \mathrm{nl}$ of saline; or 3) $100 \mu \mathrm{M}$ glutamic acid in $100 \mathrm{nl}$ of saline. After the injection the micropipette was removed, the skin was carefully sutured, and the surgical area was treated with 5\% iodine solution and ethanol. Then animal was released from stereotaxis.

Catheterization. Silicone catheters were implanted to all rats three days prior to the experiment. The proximal end of the implanted catheter was placed into $V$. jugularis externa dextra or intraperitoneal space. For intravenous catheter, slight skin dissection $(0.5-1.0 \mathrm{~cm})$ was made in right supraclavicular region to expose $V$. jugularis externa. After careful dissection of the vessel it was surrounded by two ligatures and the distal one was tightened. Specially made silicone catheter with fixator was introduced into the vein through a small incision and fixed to vessel wall with additional ligatures. To prevent thrombogenesis each catheter was filled with sterile heparin solution $(100 \mathrm{U} / \mathrm{ml})$ before its introduction into the vein. For intraperitoneal implantation, midline laparotomy was made and the catheter was introduced through the aperture $1.5-2 \mathrm{~cm}$ into the abdominal cavity. Peritoneum edges were stitched with blanket sutures. Distal end of each catheter was deduced on dorsal surface of rat's head through subcutaneous "tunnel" and fixed with ligatures. Skin was stitched with single interrupted sutures.

Experimental protocol. To diminish the possible effect of circadian rhythms on the recorded data, all experiments were conducted at fixed time period-from 8 till 9 a.m. At the beginning of the experiment, rats were placed in compact boxes to limit their mobility. All rats were preliminary habituated to spend $3-4$ hours in similar boxes according to the method described elsewhere [28, 29]. Boxes were placed in thermostat, and the temperature was raised to $29^{\circ} \mathrm{C}-30^{\circ} \mathrm{C}$ within 1 hour. The humidity level in thermostat was maintained at $50 \%$.

Copper-constantan thermocouples were used to record deep $\left(\mathrm{T}_{\mathrm{r}}\right)$ body temperature (electric thermometer "Physitemp", USA). Hot junction of each thermocouple was introduced into animals' rectum $7 \mathrm{~cm}$ in depth from anus.

1.5 hours after the adaptation of animals to experimental conditions control measurements of recorded parameters were made (twice with 30 min interval). Injection of endotoxin $(3 \mu \mathrm{g} / \mathrm{kg}$, lipopolysaccharide, LPS, Escherichia coli, 0111:B4, List Biological Laboratories, Campbell, CA; lot No LPS-25E) or apyrogenic saline (APS, Abbott Laboratories, North Chicago, IL 60,064; lot No 18-379-DK) was performed intraperitoneally of intravenously through silicone catheter in the amount not exceeding $0.5 \mathrm{ml}$. $\mathrm{T}_{\mathrm{r}}$ monitoring was continuously carried out for at least 7 hours after the injection.

Both absolute value and deviations from the average values of $T_{r}$ were registered at rest before injection and taken as the initial value for statistical analysis. Data plotting was done using "Origin 6.0". Statistical data analysis was made using the Student t-test and ANOVA program of variation statistics. Results after statistical analysis are presented in text and figures as $M \pm m$.

\section{RESULTS}

Injections of apyrogenic saline did not induce significant 
changes in $T_{\mathrm{r}}$ in any experimental group. Intravenous injection of $3 \mu \mathrm{g} / \mathrm{kg} \mathrm{E}$. coli LPS to the rats which received $100 \mathrm{nl}$ of saline into caudal part of NST was followed by an increase in rectal temperature. The increase reached $1.3^{\circ} \mathrm{C} \pm 0.2^{\circ} \mathrm{C}(\mathrm{F}=39 ; \mathrm{P}=0.003)$ by 75 th minute of observation $\left(\mathrm{T}_{\mathrm{r}}\right.$ initial values were $\left.38.0^{\circ} \mathrm{C} \pm 0.1^{\circ} \mathrm{C}\right)$. Initial values restored by 300th - 330th minute after the injection (Figure 1).

In the animals, which received kainic acid into caudal part of NST LPS produced a significantly smaller wave of $\mathrm{T}_{\mathrm{r}}$ rise. The highest elevation from the baseline level $\left(37.9^{\circ} \mathrm{C} \pm 0.1^{\circ} \mathrm{C}\right)$ was $0.6^{\circ} \mathrm{C} \pm 0.2^{\circ} \mathrm{C}$ by 90 th minute after the injection (Figure 1).

In the rats, which received $100 \mathrm{nl}$ of saline into the rostral part of NST, injection of LPS induced an increase in $T_{\mathrm{r}}$ starting from 30th minute after the injection (Figure 2). The rise of $\mathrm{T}_{\mathrm{r}}$ reached $1.3^{\circ} \pm 0.2^{\circ}$ by 75 th minute of observation $(\mathrm{F}=32.5 ; \mathrm{P}=0.001)$. The temperature returned to baseline level by 330th - 350th minute (Figure 2). Animals, which received kainic acid into the same part of NST demonstrated a pronounced biphasic temperature response to LPS. The first increase of $T_{r}$ reached its maximum at 90 minutes and was $1.44^{\circ} \mathrm{C} \pm$ $0.16^{\circ} \mathrm{C}$ higher than initial level; the second increase was at 240 minutes $\left(1.34^{\circ} \mathrm{C} \pm 0.16^{\circ} \mathrm{C}\right)$ (Figure 2).

The fact that at the late stages after intravenous injection of endotoxin the increase of colonic temperature was significantly higher in neurotoxin-treated rats $(\mathrm{F}=8.8 ; \mathrm{P}$ $<0.05$ ) corresponds to the literature $[4,5]$.

Interestingly, rats, which received neurotoxin microinjection into caudal part of NST did not demonstrate an increase of colonic temperature after intraperitoneal injection of $3 \mu \mathrm{g} / \mathrm{kg}$ LPS (Figure 3).

Rats, which received glutamate or saline into caudal parts of VLM showed an increase in $T_{r}$ after the LPS injection (Figure 4). In glutamate-treated group the increase

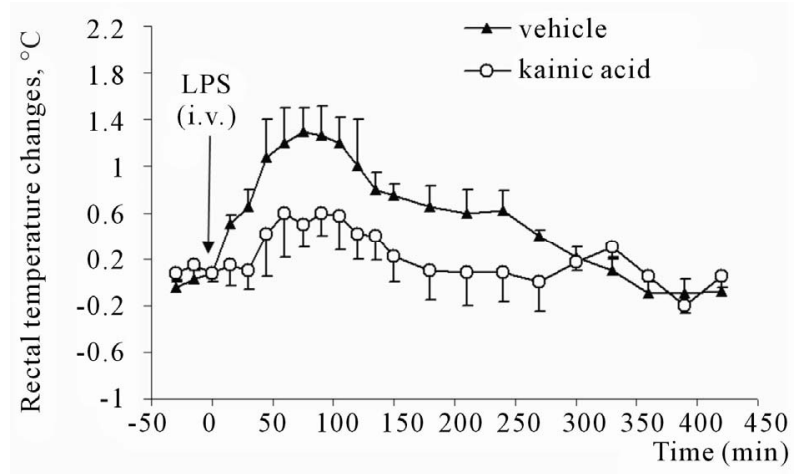

Figure 1. Rectal temperature changes in rats after intravenous injection of E. coli lipopolysaccharide ( $3 \mu \mathrm{g} / \mathrm{kg})$. Animals previously ( 28 days before the experiment) received microinjection of kainic acid $(0.5 \mu \mathrm{g}$ in $100 \mathrm{nl})$ or vehicle (saline, $100 \mathrm{nl}$ ) into caudal part of commissural region of nucleus of solitary tract.

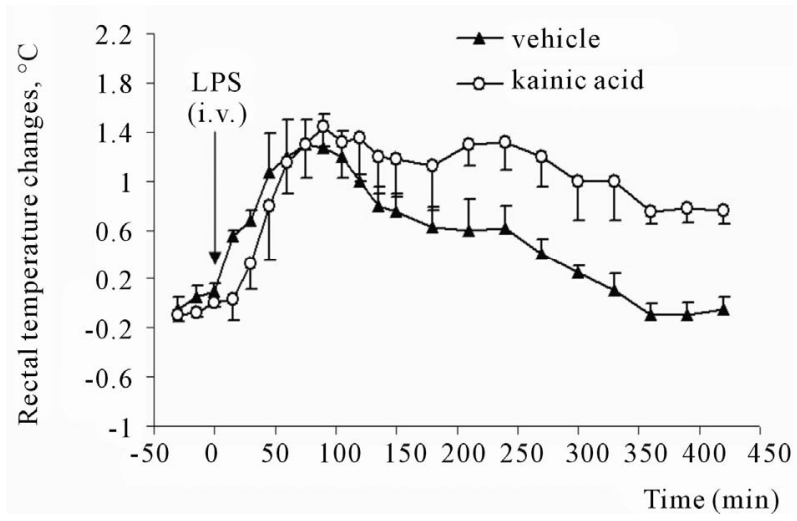

Figure 2. Rectal temperature changes in rats after intravenous injection of $E$. coli lipopolysaccharide $(3 \mu \mathrm{g} / \mathrm{kg}$ ). Animals previously (28 days before the experiment) received microinjection of kainic acid $(0.5 \mu \mathrm{g}$ in $100 \mathrm{nl})$ or vehicle (saline, $100 \mathrm{nl}$ ) into rostral part of commissural region of nucleus of solitary tract.

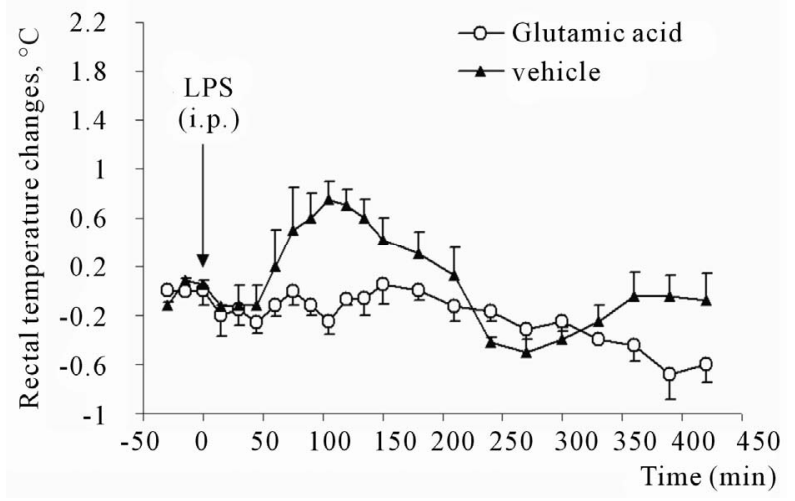

Figure 3. Rectal temperature changes in rats after intraperitoneal injection of $E$. coli lipopolysaccharide $(3 \mu \mathrm{g} / \mathrm{kg})$. Animals previously (28 days before the experiment) received microinjection of glutamic acid $(0.5 \mu \mathrm{M}$ in $100 \mathrm{nl})$ or vehicle (saline, $100 \mathrm{nl}$ ) into caudal part of commissural region of nucleus of solitary tract.

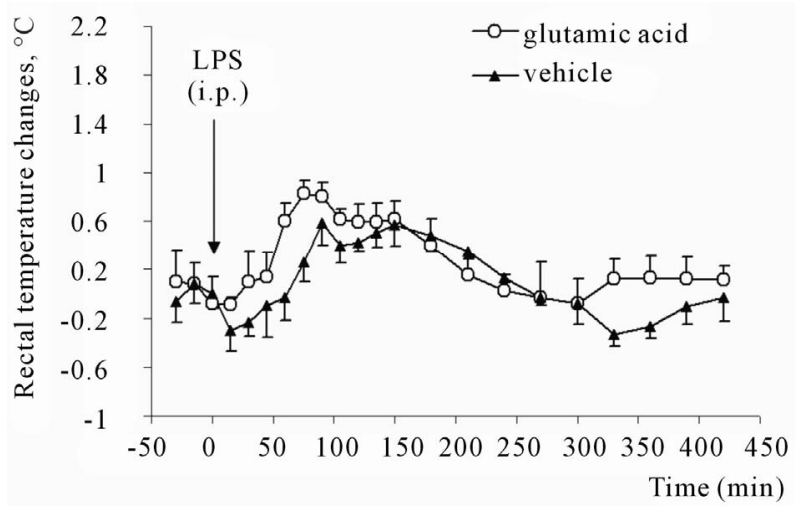

Figure 4. Rectal temperature changes in rats after intraperitoneal injection of $E$. coli lipopolysaccharide ( $3 \mu \mathrm{g} / \mathrm{kg})$. Animals previously (28 days before the experiment) received microinjection of glutamic acid $(0.5 \mu \mathrm{M}$ in $100 \mathrm{nl})$ or vehicle (saline, $100 \mathrm{nl}$ ) into caudal parts of ventrolateral medulla. 
reached $0.83^{\circ} \mathrm{C} \pm 0.1^{\circ} \mathrm{C}$ (from the initial level of $38.0^{\circ} \mathrm{C}$ ) by 75 th minute after the injection and was significantly higher than in control animals $(\mathrm{F}=10.5 ; \mathrm{P}=0.02)$.

\section{DISCUSSION}

Our findings indicate that even partial destruction of bulbar neuronal populations in the projection zones of vagus nerves can have a strong effect on the thermal responses to the systemic E. coli LPS. These facts correspond with current scientific views on the subject. Under endotoxemia hypothetical mediators causing the interaction between nervous and immune system (cytokines, prostaglandins, nitrogen monoxide [1-6]) are able to penetrate into the brain in the area of circumventricular organs, for example in area postrema, and induce the acute phase reaction [15]. Fast expression of c-fos proteins in NST, hypothalamus, dorsomedial and ventrolateral parts of brainstem and also in all circumventricular organs confirm this. Consequently, after the supposed penetration of so-called acute phase reaction messengers into the brain and area postrema the conditions for their direct contact with NST neurons are formed. So, the interaction of hormone and immune systems is implemented after the detection of infection at the level of dorsal vagal complex [15]. It is confirmed by the data on the local production of vasopressin (that changes the vascular tone, affects learning and memory processes [30]), corticosterone (that prevents inflammatory and metabolic disturbances, caused by pro-inflammatory cytokines [4]) and leptin (that is involved in the regulation of energy homeostasis, participates in systemic inflammatory response development under fever or hypothermia and anorexia, changes locomotor behavior in animals, especially under endotoxemia [15]). These facts confirm the presence of feedback channel between brain and immune system $[4,6]$. It is shown that specific antibodies are produced in dorsal motor nucleus in response to vagus nerve activation by pro-inflammatory substances $[6,15]$. This allows autonomous nervous system to regulate deep body temperature (reaction of local vasodilation in the internal organs and vasoconstriction of skin blood vessels contribute to body temperature elevation and increase in glucose uptake by muscle tissue [31]) in response to changes in hormone and immune status of the organism under endotoxemia.

Local destruction of neurons in rostral or caudal parts of NST is accompanied with violation in central mechanisms responsible for protective reactions formation, in particular the regulation of deep body temperature during endotoxin action in the organism. Noticeably, the responses to LPS did not depend on the method of injecttion (intravenous or intraperitoneal), but was determined by determined by topographic orientation of regions in NST which received neurotoxin. Despite the small volume of solutions injected into NST (100 nl) we cannot eliminate the possibility of neurotoxins diffusion into brain areas surrounding the site of microinjection. Theoretical calculation shows that the injection of $100 \mathrm{nl}$ of solution forms a sphere with diameter of $0.7 \mathrm{~mm}$. Taking into account this view it should be stated that the actual results of intracentral microinjections strikingly differ during neurotoxins injection into NST parts separated from each other only by $200-500 \mu \mathrm{m}$, although this distance is much shorter than the theoretically possible diffusion paths. Presumably this fact can be explained not only by morphofunctional heterogeneity of different parts of NST, but also unequal representation of neuronal elements of the system, responsible for deep body temperature control, in dorsal parts of VLM. It is also known that NST may affect the balance of heat production and loss by regulation of cryogenic factors release, such as arginine-vasopressin $[4,32]$. The confirmation can be found in several studies $[30,32]$. It is also known that hypothalamic neuropeptide vasopressin improves the processes of consolidation and delays the extinction of conditioned reflexes [30,32], is spread in axonal way to many brain structures, including hippocampus, and may influence memory and learning processes.

Caudal parts of VLM has close synaptic contacts with NST [12,19]. Neurons of these nuclei transmit afferent signals from NST to sympatoactive neurons of rostral parts of VLM and to neurosecretory hypothalamic cells $[12,19]$. The significant amplification of the temperature response to LPS in rats, which received glutamate into caudal parts of VLM can be explained by the presence of capsaicin-sensitive area in this brain region $[12,15]$.

Taken together, these results demonstrate that even partial destruction in central vagal projections can lead to the significant changes in the thermoregulatory reactions to endotoxemia. We suppose that this fact has importance for clinical practice, since damage of peripheral nerve fibres are known to provoke degeneration in their central projections and associated neuronal populations.

\section{REFERENCES}

[1] Lam, D.K., Sessle, B.J. and Hu, J.W. (2009) Glutamate and capsaicin effects on trigeminal nociception II: Activation and central sensitization in brainstem neurons with deep craniofacial afferent input. Brain Research, 1253, 48-59. doi:10.1016/j.brainres.2008.11.056

[2] Maione, S., Starowicz, K., Cristino, L., Guida, F., Palazzo, E., Luongo, L., Rossi, F., Marabese, I., de Novellis, V. and Di Marzo, V. (2009) Functional interaction between TRPV1 and mu-opioid receptors in the descending antinociceptive pathway activates glutamate transmission and induces analgesia. Journal of Neurophysiology, 101, 24112422. doi:10.1152/jn. 91225.2008

[3] Mandadi, S. and Roufogalis, B.D. (2008) ThermoTRP channels in nociceptors: Taking a lead from capsaicin re- 
ceptor TRPV1. Current Neuropharmacology, 6, 21-38. doi:10.2174/157015908783769680

[4] Meldrum, B.S. (2000) Glutamate as a neurotransmitter in the brain: Review of physiology and pathology. Journal of Nutrition, 130, 1007S-1015S.

[5] Ootsuka, Y., Blessing, W.W., Steiner, A.A. and Romanovsky, A.A. (2008) Fever response to intravenous prostaglandin E2 is mediated by the brain but does not require afferent vagal signaling. American Journal of Physiology-Regulatory, Integrative and Comparative Physiology, 294, R1294-R1303. doi:10.1152/ajpregu.00709.2007

[6] Palazzo, E., Luongo, L., de Novellis, V., Rossi, F., Marabese, I. and Maione, S. (2012) Transient receptor potential vanilloid type 1 and pain development. Current Opinion in Pharmacology, 12, 9-17. doi:10.1016/j.coph.2011.10.022

[7] Bluthe, R.-M., Walter, V., Parnet, P., Laye, S., Lestage, J., Verrier, D., Poole, S., Stenning, B.E., Kelley, K.W. and Dantzer, R. (1994) Lipopolysaccharide induces sickness behaviour in rats by a vagal mediated mechanism. Comptes Rendus de l'Académie des Sciences, 317, 499-503.

[8] Watkins, L.R., Wiertelak, E.P., Goehler, L.E., MooneyHeiberger, K., Martinez, J., Furness, L., Smith, K.P. and Maier, S.F. (1994) Neurocircuitry of illness-induced hyperalgesia. Brain Research, 639, 283-299. doi:10.1016/0006-8993(94)91742-6

[9] Watkins, L.R., Maier, S.F. and Goehler, L.E. (1995) Сytokine-to-brain communication: A review and analysis of alternative mechanisms. Life Sciences, 57, 1011-1026. doi:10.1016/0024-3205(95)02047-M

[10] Gaykema, R.P.A., Dijkstra, I. and Tilders, F.J.H. (1995) Subdiaphragmatic vagotomy suppresses endotoxin-induced activation of hypothalamic corticotropin-releasing hormone neurons and ACTH secretion. Endocrinology, 136, 4717-4720. doi:10.1210/en.136.10.4717

[11] Blatteis, C.M. and Sehic, E. (1997) Fever: How may circulating pyrogens signal the brain? News in Physiological Science, 12, 1-9.

[12] Goldbach, J.-M., Roth, J. and Zeisberger, E. (1997) Fever suppression by subdiaphragmatic vagotomy in guinea pigs depends on the route of pyrogen administration. American Journal of Physiology, 272, R675-R681.

[13] Sehic, E. and Blatteis, C.M. (1996) Blockade of lipopolysaccharide-induced fever by subdiaphragmatic vagotomy in guinea pigs. Brain Research, 726, 160-166. doi:10.1016/0006-8993(96)00326-5

[14] Simons, C.T., Kulchitsky, V.A., Sugimoto, N., Homer, L.D., SzeÂkely, M. and Romanovsky, A.A. (1998) Signaling the brain in systemic inammation: Which vagal branch is involved in fever genesis? American Journal of Physiology, 275, R63-R68.

[15] Bishnoi, M. and Premkumar, L.S. (2011) Possible consequences of blocking transient receptor potential vanilloid. Current Pharmaceutical Biotechnology, 12, 102-114. doi:10.2174/138920111793937907

[16] Forsythe, P., Kunze, W.A. and Bienenstock, J. (2012) On communication between gut microbes and the brain. Current Opinion in Gastroenterology, 28, 557-562.

\section{doi:10.1097/MOG.0b013e3283572ffa}

[17] Rahimi-Movaghar, V., Yazdi, A. and Mohammadi, M. (2008) Usefulness of the tail-flick reflex in the prognosis of functional recovery in paraplegic rats. Surgical Neurology, 70, 323-325. doi:10.1016/j.surneu.2007.05.056

[18] Koulchitsky, S.V. (1998) Are the capsaicin-sensitive structures of ventral medulla involved in the temperature response to endotoxin in rats? Neuroscience Letters, 244, 112-114. doi:10.1016/S0304-3940(98)00128-1

[19] Koulchitsky, S.V., Levkovets, V.S., Tchitchkan, D.N., Soltanov, V.V. and Kulchitsky, V.A. (1999) Role of the solitary tract nucleus and caudal ventrolateral medulla in temperature responses in endotoxemic rats. Life Sciences, 64, 37-43. doi:10.1016/S0024-3205(98)00531-1

[20] Koulchitsky, S.V. and Kulchitsky, V.A. (2001) Central and peripheral mechanisms of nociceptive reflexes in conditions of acute phase reaction. Proceedings of the $\mathrm{Na}$ tional Science Council, Republic of China, 25, 197-213.

[21] Bohus, B. and de Wied, D. (1998) The vasopressin deficient Brattleboro rats: A natural knockout model used in the search for CNS effects of vasopressin. Progress in Brain Research, 119, 555-573. doi:10.1016/S0079-6123(08)61593-9

[22] Lipski, J., Lin, J., Teo, M.Y. and van Wyk, M. (2002) The network vs. pacemaker theory of the activity of RVL presympathetic neurons-A comparison with another putative pacemaker system. Autonomic Neuroscience: Basic and Clinical, 98, 85-89. doi:10.1016/S1566-0702(02)00038-3

[23] Moult, P.R. and Harvey, J. (2009) Regulation of glutamate receptor trafficking by leptin. Biochemical Society Transactions, 37, 1364-1368. doi:10.1042/BST0371364

[24] Herman, M.A., Alayan, A., Sahibzada, N., Bayer, B., Verbalis, J., Dretchen, K.L. and Gillis, R.A. (2010) micro-Opioid receptor stimulation in the medial subnucleus of the tractus solitarius inhibits gastric tone and motility by reducing local GABA activity. American Journal of Physiology-Gastrointestinal and Liver Physiology, 299, G494-G506. doi:10.1152/ajpgi.00038.2010

[25] Holden, J.E. and Pizzi, J.A. (2008) Lateral hypothalamic-induced antinociception may be mediated by a substance P connection with the rostral ventromedial medulla. Brain Research, 1214, 40-49. doi:10.1016/j.brainres.2008.03.051

[26] Koulchitsky, S.V., Azev, O.A., Gourine, A.V. and Kulchitsky, V.A. (1994) Capsaicin-sensitive area in the ventral surface of the rat medulla. Neuroscience Letters, 182, 129-132. doi:10.1016/0304-3940(94)90780-3

[27] Paxinos, Y. and Watson, C. (1986) The Rat Brain in stereotaxic coordinates. Academic Press, Orlando.

[28] Goncharuk, V.D., Buijs, R.M., Jhamandas, J.H. and Swaab, D.F. (2011) Vasopressin (VP) and neuropeptide FF (NPFF) systems in the normal and hypertensive human brainstem. Journal of Comparative Neurology, 519, 93124. doi: $10.1002 / \mathrm{cne} .22507$

[29] Kozak, W., Kluger, M.J., Tesfaigzi, J., Kozak, A., Mayfield, K.P., Wachulec, M. and Dokladny, K. (2000) Molecular mechanisms of fever and endogenous antipyresis. 
Annals of New York Academy of Sciences, 917, 121-134. doi:10.1111/j.1749-6632.2000.tb05376.x

[30] Yue, C., Ma, B., Zhao, Y., Li, Q. and Li, J. (2012) Lipopolysaccharide-induced bacterial translocation is intestine site-specific and associates with intestinal mucosal inflammation. Inflammation, 35, 1880-1888. doi:10.1007/s10753-012-9510-1

[31] Yagi, S., Takaki, A., Hori, T. and Sugimachi, K. (2002) Enteric lipopolysaccharide raises plasma IL-6 levels in the hepatoportal vein during non-inflammatory stress in the rat. Fukuoka Igaku Zasshi, 93, 38-51.

[32] Wan, S., Browning, K.N., Coleman, F.H., Sutton, G., Zheng, H., Butler, A., Berthoud, H.R. and Travagli R.A. (2008) Presynaptic melanocortin-4 receptors on vagal afferent fibers modulate the excitability of rat nucleus tractus solitarius neurons. Journal of Neuroscience, 28, 49574966. doi:10.1523/JNEUROSCI.5398-07.2008 